\title{
Indispensable role of the Ubiquitin-fold modifier 1-specific E3 ligase in maintaining intestinal homeostasis and controlling gut inflammation
}

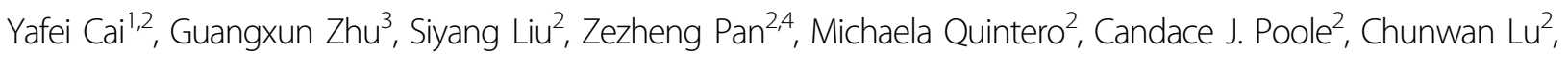 \\ Huabin Zhu' ${ }^{2}$ Bianca Islam², Jan van Riggelen², Darren Browning ${ }^{2}$, Kebin Liu², Richard Blumberg', \\ Nagendra Singh ${ }^{2}$ and Honglin $\mathrm{Li}^{2,6}$
}

\begin{abstract}
Intestinal exocrine secretory cells, including Paneth and goblet cells, have a pivotal role in intestinal barrier function and mucosal immunity. Dysfunction of these cells may lead to the pathogenesis of human diseases such as inflammatory bowel disease (IBD). Therefore, identification and elucidation of key molecular mechanisms that regulate the development and function of these exocrine cells would be crucial for understanding of disease pathogenesis and discovery of new therapeutic targets. The Ufm1 conjugation system is a novel ubiquitin-like modification system that consists of Ufm1 (Ubiquitin modifier 1), Uba5 (Ufm1-activating enzyme, E1), Ufc1 (Ufm1-conjugating enzyme, E2) and poorly characterized Ufm1 E3 ligase(s). Recent mouse genetic studies have demonstrated its indispensable role in embryonic development and hematopoiesis. Yet its role in other tissues and organs remains poorly defined. In this study, we found that both Uf1 and Ufbp1, two key components of the Ufm1 E3 ligase, were highly expressed in the intestinal exocrine cells. Ablation of either Ufl1 and Ufbp1 led to significant loss of both Paneth and goblet cells, which in turn resulted in dysbiotic microbiota and increased susceptibility to experimentally induced colitis. At the cellular and molecular levels, Ufbp 1 deficiency caused elevation of endoplasmic reticulum stress and activation of the Unfolded Protein Response (UPR) and cell death program. Administration of small molecular chaperone partially prevented loss of Paneth cells caused by acute Ufbp1 deletion. Taken together, our results have provided unambiguous evidence for the crucial role of the Ufm1 E3 ligase in maintenance of intestinal homeostasis and protection from inflammatory diseases.
\end{abstract}

\section{Introduction}

The intestinal epithelium is composed of a single layer of epithelial cells that are rapidly renewed through proliferation and differentiation of intestinal stem cells

\footnotetext{
Correspondence: Yafei Cai (ycai@njau.edu.cn) or Honglin Li (hli@augusta.edu) ${ }^{1}$ College of Animal Science and Technology, Nanjing Agricultural University, 210095 Nanjing, Jiangsu, China

2Department of Biochemistry \& Molecular Biology, Georgia Cancer Center, Medical College of Georgia, Augusta University, Augusta, GA 30912, USA Full list of author information is available at the end of the article.

These authors contributed equally: Yafei Cai, Guangxun Zhu
}

(ISCs). In addition to digestion and absorption of nutrients, the intestinal epithelium also serves as a defensive barrier to prevent against infection of gut pathogens. Intestinal epithelial cells (IECs) consist of stem cells, proliferating progenitor cells and highly differentiated absorptive and secretory cells. Whereas absorptive enterocytes are responsible for nutrient absorption, professional secretory cells such as Paneth and goblet cells have a crucial role in maintaining intestinal homeostasis and mucosal immunity. Goblet cells synthesize and

\section{(c) The Author(s) 2019}

(c) (i) Open Access This article is licensed under a Creative Commons Attribution 4.0 International License, which permits use, sharing, adaptation, distribution and reproduction cc) in any medium or format, as long as you give appropriate credit to the original author(s) and the source, provide a link to the Creative Commons license, and indicate if changes were made. The images or other third party material in this article are included in the article's Creative Commons license, unless indicated otherwise in a credit line to the material. If material is not included in the article's Creative Commons license and your intended use is not permitted by statutory regulation or exceeds the permitted use, you will need to obtain permission directly from the copyright holder. To view a copy of this license, visit http://creativecommons.org/licenses/by/4.0/. 
secrete mucin proteins, the major component of intestinal mucus that protects the epithelium from bacterial infection ${ }^{1}$. Paneth cells are responsible for synthesis and secretion of a large quantity of antimicrobial peptides that maintain homeostatic microbiota in the gut ${ }^{2,3}$. They also have a crucial role in the formation of ISC niche and regulation of self-renewal and differentiation of ISCs under conditions such as calorie restriction ${ }^{4,5}$. A breakdown of intestinal tissue homeostasis may lead to human diseases such as inflammatory bowel disease (IBD). Loss of Paneth cells or impairment of their normal function is often observed in the IBD patients and contributes to disease pathogenesis ${ }^{6,7}$. Goblet cell deficiency or dysfunction is also causative for ulcerative colitis $(\mathrm{UC})^{8,9}$. Therefore, elucidation of the underlying mechanism that governs development, survival and function of Paneth and goblet cells would be crucial for development of novel therapies for inflammatory diseases.

Post-translational modification of target proteins by Ubiquitin (Ub) and Ubiquitin-like (Ubl) modifiers has important roles in multiple cellular processes, and dysfunction of this process has been implicated in the pathogenesis of a variety of human diseases ${ }^{10}$. Ufm 1 (Ubiquitin-fold modifier $\underline{1}$ ) is a small Ubl modifier with 85 amino acid residues and highly conserved in multicellular organisms ${ }^{11}$. With a limited primary sequence identity with $\mathrm{Ub}$ and other Ubls, Ufm1 displays a solution structure of ubiquitin-fold that is shared by other Ubls ${ }^{12}$. However, unlike Ub and some Ubls with di-glycine (GG) residues at the carboxyl (C)-terminus of their active forms, the active Ufm1 contains Valine-Glycine (VG) residues at its $\mathrm{C}$-terminus, and its Glycine residue is covalently conjugated to the lysine residues of target proteins via an iso-peptide bond. Ufm1 conjugation to its target proteins, a process termed as ufmylation, is accomplished in multi-step biochemical reactions that are catalyzed by a set of Ufm1-specific enzymes, namely, Ufm1-activating E1 enzyme Uba5, Ufm1-conjugating E2 enzyme Ufc1, and Ufm1-specific E3 ligase(s) ${ }^{11}$. However, the identity of Ufm 1 targets and the mechanism to control this conjugating process is poorly understood.

Although Ufm1-sepcific E1 (Uba5) and E2 (Ufc1) enzymes share signature domains with other E1s and E2s, only one Ufm1-specific E3 ligase has been identified so far and this E3 ligase does not contain any conventional E3 ligase domains or motifs that mediate ubiquitination or ubiquitination-like modifications. Ufl1 (Ufm1 ligase $\underline{1}$, as known as KIAA0776, RCAD, NLBP and Maxer) was originally isolated by several independent studies as a novel Cdk5rap3-binding protein and regulator of NF- $k B$ signaling ${ }^{13-15}$. Interestingly, Tatsumi et al. found that Ufl1 promoted ufmylation of Ufbp1 ( $\underline{\mathrm{Ufm} 1}$ binding protein $\underline{1}$, also named as DDRGK1, C20orf116 and Dashurin), an endoplasmic reticulum (ER)-anchored Ufl1-binding protein $^{14,16,17}$. This study provided biochemical evidence for the notion that Ufl1 may function as a Ufm1-specific E3 ligase. Yoo et al. subsequently found that Ufl1 also promoted ufmylation of ASC1 (Activating Signal Cointegrator 1, also known as TRIP4), a transcription coactivator of nuclear receptors ${ }^{18,19}$. In breast cancer cells, ufmylated ASC1 serves as a platform to facilitate recruitment of estrogen receptor and other transcription co-activators and enhance transcription of target genes, thereby promoting breast cancer development ${ }^{19}$. Interestingly, ufmylated Ufbp1 functions as a co-factor in the process of ASC1 ufmylation ${ }^{19}$.

Since its discovery more than a decade ago, the physiological function of the Ufm1 conjugation system has remained unclear until very recently. Genetic studies using gene knockout (KO) mouse models have provided undisputable evidence for its essential role in animal development. Germline deletion of individual ufmylation genes, including Uba5, Ufl1 and Ufbp1, led to impaired red blood cell development and embryonic lethality around E11.5-E13.5 days ${ }^{20-22}$. Acute ablation of either Ufl1 or Ufbp 1 in adult mice caused pancytopenia and severe anemia, further highlighting the indispensable role of ufmyation in erythropoiesis ${ }^{20-22}$. In addition to its role in animal development, multiple lines of evidence indicate involvement of the ufmylation pathway in the pathogenesis of various human dieseases, including hematopoietic diseases $^{23,24}$, diabetes $^{25}$, ischemic heart injury ${ }^{26}$, skeletal dysplasia $^{27}$, atherosclerosis ${ }^{28}$, and cancer ${ }^{19,29}$. Intriguingly, variants of human Ufm1, Uba5 and Ufc1 genes have been found to be linked to early-onset encephalopathy and defective brain development ${ }^{30-35}$, while human $U f b p 1$ was identified as one of the new genetic risk loci in Parkinson's disease ${ }^{36}$, indicating a crucial role of ufmylation in neural development and function. Furthermore, loss-of-function mutation in $U f b p 1$ gene was found to be a causative factor in Shohat-type spondyloepimetaphyseal dysplasia (SEMD), a skeletal dysplasia that affects cartilage development $^{37}$. Mutations in Ufm1-specific protease Ufsp2 were found in the patients with Beukes hip dysplasia ${ }^{27}$. Taken together, these recent findings underscore the importance of the Ufm1 system in normal development and physiology. Nonetheless, the full scope of its biological function and involvement in disease pathogenesis remains to be further defined.

During the course of characterizing Ufl1 and Lfbp 1 conditional knockout mice, we frequently observed gut bleeding in some conditional knockout mice, and this observation prompted us to investigate the potential role of these genes in the intestine. Using both acute and tissue-specific knockout mouse models, we found that ablation of either Ufl1 or Ufbpl led to significant loss of Paneth and goblet cells, which in turn resulted in dysbiotic microbiota and increased susceptibility to 


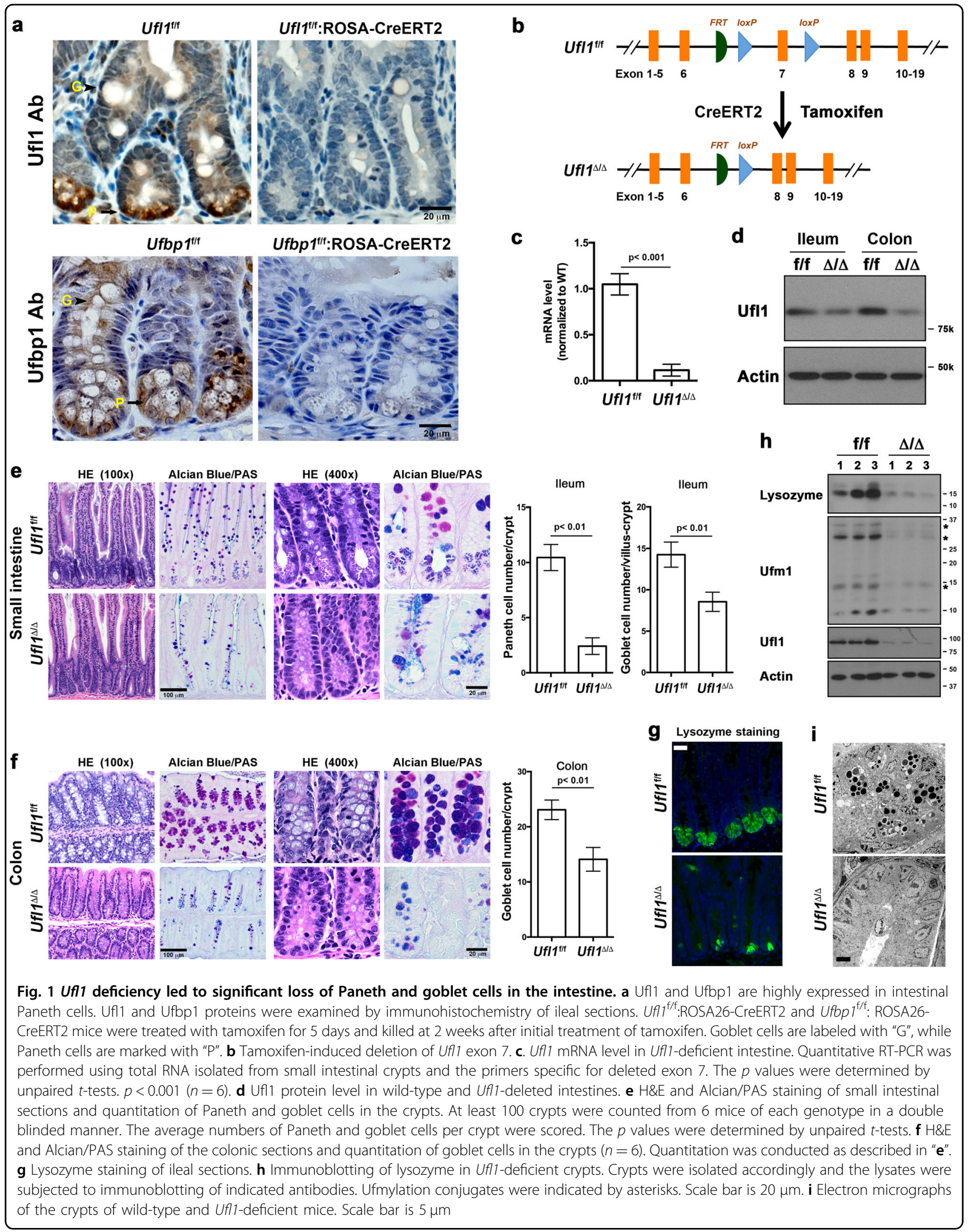




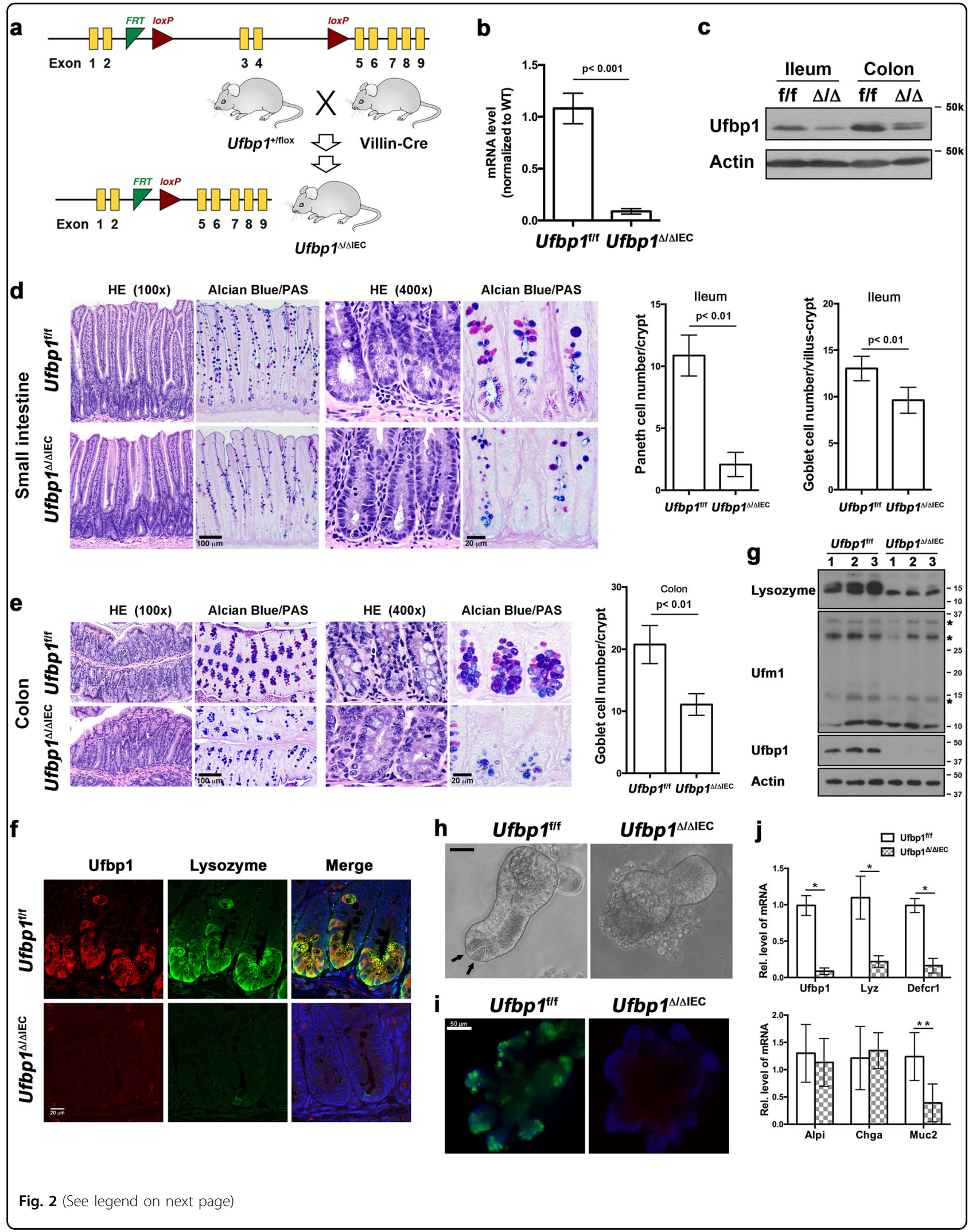




\begin{abstract}
(see figure on previous page)
Fig. 2 IEC-specific deletion of Ufbp 1 resulted in depletion of Paneth and goblet cells. a Ufbp 1 floxed allele and generation of IEC-specific Ufbp 1 knockout mice. b Ufbp1 mRNA level in Ufbp 1-deficient intestine. Quantitative RT-PCR was performed using total small intestinal crypt RNA and the primers specific for deleted exons 3 and 4. The $p$ values were determined by unpaired $t$-tests $p<0.001(n=6)$. c Ufbp1 protein level in Ufbp $1^{\Delta / \Delta \mathrm{EEC}}$ intestine. $\mathbf{d} \mathrm{H} \& \mathrm{E}$ and Alcian/PAS staining of the small intestinal sections and quantitation of Paneth and goblet cells in Ufbp $1^{\Delta \Delta \Delta \mathrm{EC}}$ mice. At least 100 crypts were counted from 6 mice of each genotype in a double blinded manner. The average numbers of Paneth and goblet cells per crypt were scored. The $p$ values were determined by unpaired $t$-tests. e H\&E and Alcian/PAS staining of the colonic sections and quantitation of goblet cells in the crypts $(n=6)$. $\mathbf{f}$ Double immunostaining of ileal sections using Ufbp1 and lysozyme antibodies. $\mathbf{g}$ Immunoblotting of lysozyme in Ufl1-deficient crypts. Crypts were isolated accordingly and the lysates were subjected to immunoblotting of indicated antibodies. Ufmylation conjugates were indicated by asterisks. $\mathbf{h}$ In vitro organoid culture of wild-type and Ufbp 1-deficient crypts. Paneth cells in the wild-type organoid were marked by arrows. Scale bar is $50 \mu \mathrm{m}$. i Lysozyme immunostaining of cultured organoid. $\mathbf{j}$ Quantitative RT-PCR analysis of gene expression using total RNA isolated from culture organoids. Six independent cultures for each genotype were used for the analysis. The $p$ values were determined by unpaired $t$ tests. ${ }^{*} p<0.001,{ }^{* *} p<0.01(n=6)$. Lyz lysozyme; Defcr1 defensin a1; Alpi alkaline phosphatase; Chga Chromogranin A; and Muc2 mucin 2
\end{abstract}

experimentally induced colitis. Our findings have identified the ufmylation pathway as a novel molecular mechanism to control intestinal homeostasis.

\section{Results}

Acute ablation of Ufl1 caused significant loss of Paneth and goblet cells in the intestine

We first examined expression of Ufl1 and Ufbp1 in the IECs. Both Ufl1 and Ufbp1 are present in all types of IECs, and highly expressed in exocrine Paneth cells (Fig. 1a). Antibody specificity was confirmed by lack of staining in IECs with tamoxifen-induced deletion of each gene, respectively (Fig. 1a). To investigate the function of Ufl1 in the intestine, we acutely ablated Lfl 1 in adult Ufl1 floxed mice (Ufl1 ${ }^{\mathrm{f} / \mathrm{f}}:$ ROSA26-CreERT2) by tamoxifen administration (Fig. 1b). Deletion of Ufl1 allele in the gut was confirmed by quantitative RT-PCR using primers specific for the deleted exon 7 (Fig. 1c), immunoblotting of Ufl1 protein (Fig. 1d, h) and immunohistochemistry (Fig. 1a). Although the gross anatomy of the intestinal epithelium was not compromised in Ufl1-deleted mice, close examination of intestinal sections revealed substantial loss of both Paneth and goblet cells in the crypts of Ufl1-deficient ileum (Fig. 1e) and goblet cells in the colon (Fig. 1f). The result was further confirmed by Alcian blue/Periodic acid-Schiff (PAS) staining (Fig.1e, f), which specifically marks secretory cells such as Paneth and goblet cells. Loss of Paneth cells in the small intestine was also confirmed by reduced staining of lysozyme, an early Paneth cell-specific marker (Fig. 1g). Decreased amount of lysozyme in Ufl1-deficient intestine was further demonstrated by immunoblotting of lysozyme in crypt lysates (Fig. 1h). To exclude the possibility that $U f l 1$ deficiency may impair lysozyme synthesis which may mimic Paneth cell loss, we performed electron microscopy (EM) of the crypts. In comparison to wild-type intestinal crypts that contained many Paneth cells with extensive ER network and secretory granules, Ufl1-deficient crypts had very few cells with a rich ER network, indicating the absence of Paneth cells (Fig. 1i). Taken together, these results strongly suggest that Ufl1 is crucial for intestinal exocrine cells.

\section{IEC-specific knockout of Ufbp1 led to depletion of Paneth and goblet cells}

In addition to its putative role as a Ufm1-specific E3 ligase, Ufl1 is also involved in regulating the stability of its interacting proteins, C53 and Ufbp1, in a Ufm1independent manner ${ }^{14,16}$. Ufbp1 is a Ufm1 substrate and a co-factor of Ufm1 E3 ligase ${ }^{16,19}$, and Ufbp1 null mice exhibit the phenotype similar to the one of $U f l 1$ knockout mice with defective embyronic development and impaired hematopoiesis ${ }^{21,22}$. To further identify Ufl1's downstream effector and define their functional role in the gut, we therefore generated Ufbp 1 IEC-specific knockout model using Villin-Cre transgenic mice (Fig. 2a) ${ }^{38}$. Deletion of exons 3 and 4 resulted in loss of Ufbp1 protein in the intestine (Fig. 2b, c, g). Similar to Ufl1 conditional KO mice, IEC-specific deletion of $U f b p 1\left(U f b p 1^{\Delta / \Delta \mathrm{IEC}}\right)$ led to a significant loss of Paneth and goblet cells in the intestinal epithelium (Fig. 2d-f). Paneth cell loss was further confirmed by decreased expression of Paneth cell-specific genes, such as lysozyme (Fig. 2g), Defcr 1 and 5 (Supplementary Fig. S1a). Interestingly, the number of enteroneuroendocrine cells (marked by Chromogranin A) was not affected by $U f b p 1$ deficiency (Supplementary Fig. S1b). Although isolated Ufbp1-deficient crypts were able to grow and develop into mature organoids (Fig. 2h), these cultured organoids did not contain Paneth cells and their absence was further validated by lack of lysozyme staining and reduction of Paneth cell-specific gene expression (Fig. $2 \mathrm{~h}-\mathrm{j}$ ). Together, our results demonstrate that like Ufl1, Ufbp1 is also essential for intestinal exocrine cells.

\section{Paneth cell-specific deletion of Ufbp 1 resulted in the decrease of Paneth cells}

Intestinal stem cells are responsible for renewal of intestinal epithelial cells including Paneth and goblet cells. It remained unclear whether loss of exocrine lineage in 

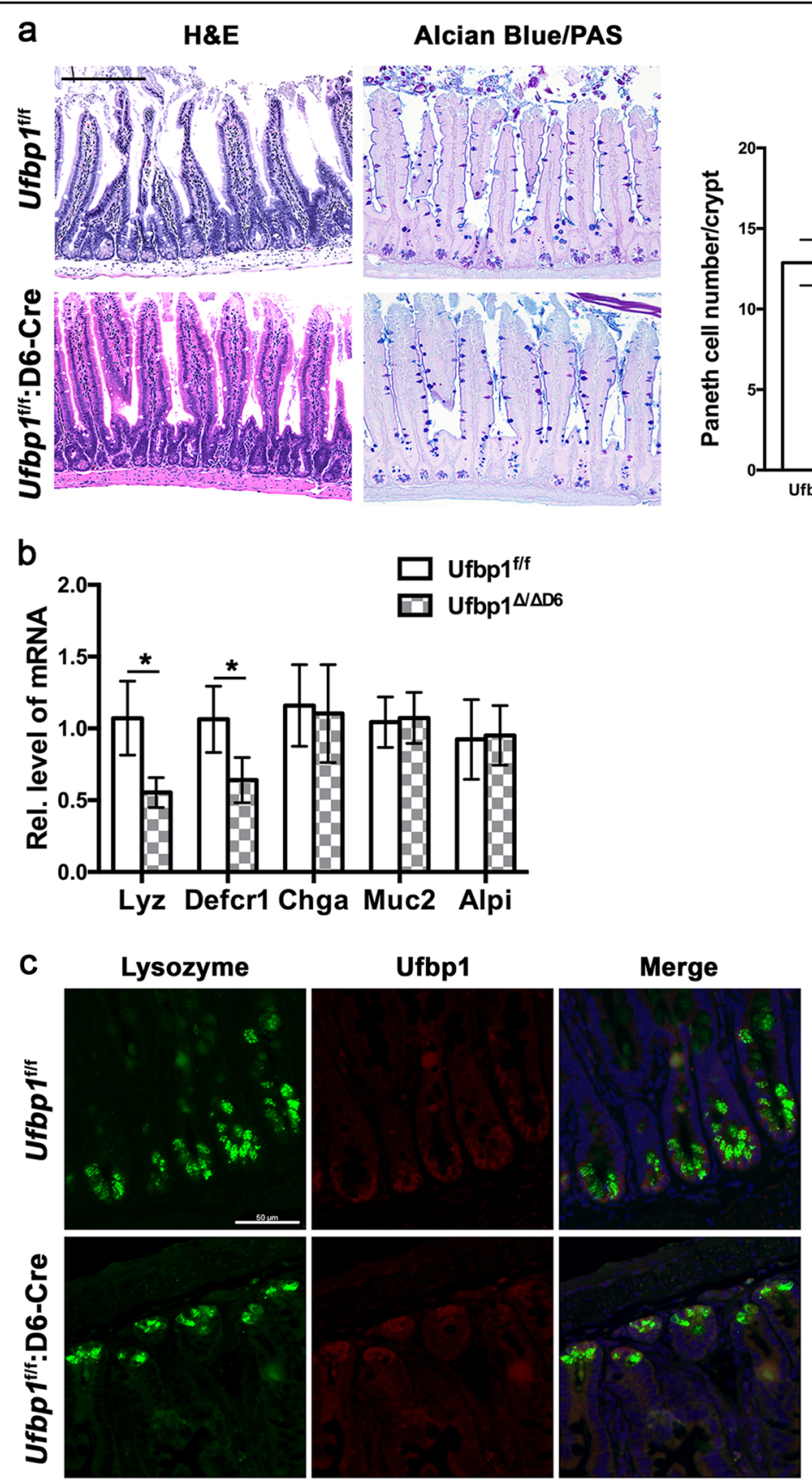

Fig. 3 Paneth cell-specific ablation of Ufbp1 led to the decrease of Paneth cells. a H\&E and Alcian/PAS staining of small intestinal sections and quantitation of Paneth cells in the crypts. At least 100 crypts were counted from 6 mice of each genotype in a double blinded manner. The average numbers of Paneth cells per crypt were scored. The $p$ value was determined by unpaired $t$-tests. Scale bar is $100 \mu m$. $\mathbf{b}$ Quantitative RT-PCR analysis of Paneth cell gene expression using total RNA isolated from ileal crypts. Crypts were isolated from six mice of each genotype. The $p$ values were determined by unpaired $t$-tests. ${ }^{*} p<0.01(n=6)$. c Double immunostaining of ileal sections using Ufbp1 and lysozyme antibodies. Scale bar is $50 \mu m$

Ufbp1- or Ufl1-deficient intestine is caused by impaired lineage determination of ISCs, or defective development and maturation of Paneth and goblet cells, or both. To address this question, we took advantage of Paneth cellspecific Cre transgenic mouse line in which Cre expression is under the control of defensin $\alpha 6$ (D6) promoter $^{6}$, and created Paneth cell-specific knockout mice of Ufbp1. As shown in Fig. 3a, Paneth cell-specific ablation of $U f b p 1$ led to fewer Paneth cells in the small intestine, even though the reduction was not as dramatic as in 
Ufbp $1^{\Delta / \Delta \mathrm{IEC}}$ mice (Figs. 2d and 3a). The decrease of Paneth cells was further confirmed by quantitative RTPCR analysis of Paneth cell-specific gene expression. Expression of lysozyme and defensin $\alpha 1$ was significantly reduced to half of wild-type level, while expression of chromogranin A (enteroendocrine cell marker), mucin 2 (goblet cell marker) and intestinal alkaline phosphatase (enterocyte marker) remained unchanged in Paneth cellspecific Ufbp1 KO mice (Fig. 3b). Although the role of Ufbp1 in ISCs remains to be further defined, this result strongly suggests a cell-autonomous role of Ufbp1 in Paneth cell development and maturation after lineage commitment. The difference between two Ufbp1 KO mouse models was probably due to variable penetrance of Cre transgene expression and efficiency of Cre-mediated deletion of $U f b p 1$ allele. This explanation was supported by the observation that nearly all lysozyme-positive Paneth cells in Ufl1 $^{\mathrm{f} / \mathrm{f}}$ :D6-Cre crypts were also Ufbp1positive (Fig. 3c).

\section{Ufbp $1^{\Delta / \Delta I E C}$ mice had altered fecal microbiota and were susceptible to experimentally induced colitis}

It has been shown that dysfunction of Paneth cells in mice leads to dysbiotic microbiota and loss of intestinal homeostasis, thereby conferring susceptibility to experimentally induced colitis ${ }^{2,3,6,39,40}$. We first examined the presence of a few bacterial genera in fecal pellets of Ufbp1-deficient mice using 16s rRNA gene-based profiling. As shown in Supplementary Fig. S2, Lfbp 1 deficiency led to alteration of the fecal microbiota: significant decrease of Bifidobacterium and increase of Prevotellacae. At the age of 8-10 weeks, $U f b p 1^{\Delta / \Delta \mathrm{IEC}}$ intestine did not exhibit signs of inflammation and colitis such as increased lymphocyte infiltration and elevated expression of inflammatory cytokines (see HE staining of intestinal sections in Fig. 2d, e, and the RT-PCR result in Fig. 4e). To address whether $U f b p 1$ deficiency predisposes susceptibility to inflammatory colitis, we further tested both IEC- and Paneth cell-specific Ufbp1-deficient mice in the DSS (dextran sulfate sodium)-induced colitis model. In comparison to wild-type mice, $U f b p 1^{\Delta / \Delta I E C}$ mice exhibited accelerated weight loss (Fig. 4a), colon shrinkage (Fig. 4b), deteriorated clinical scores (Fig. 4c), severe epithelial damage including significant loss of basal crypts and massive infiltration of immune cells (Fig. 4d), and elevated expression of inflammatory cytokines IL-1 $\beta$ and IL-6 (Fig. 4e). Interestingly, Paneth cell-specific Ufbp1deficient mice $\left(U f b p 1^{\Delta / \Delta \mathrm{D} 6}\right)$ also showed significant weight loss and increased expression of inflammatory cytokines even though these animals exhibited modest colon shrinkage and worsening of clinical scores (Fig. 4c-e). Taken together, these results suggest that Ufbp1 has a critical role in preventing inflammatory colitis.

\section{Ufbp1 deficiency promoted cell death of IECs}

One possible cause for loss of exocrine lineage in Lfbp1deficient intestine is accelerated cell death of exocrine cells. As assessed by TUNEL staining, a substantial increase of cell death was observed in $U f b p 1^{\Delta / \Delta \mathrm{IEC}}$ crypts (Fig. 5a). To better characterize cell death process, we examined $U f b p 1^{f f f}:$ ROSA-CreERT2 mice in which $U f b p 1$ was acutely depleted by tamoxifen (TAM) administration. Acute ablation of $U f b p 1$ led to marked increase of dying cells with pyknotic nuclei in the crypts, and the dying cells appeared to be Paneth and goblet cells (Fig. 5b). The peak of cell death usually occurred at 2 weeks after initiation of tamoxifen treatment (Fig. 5c). Active caspase-3 positivity was observed in Ufbp1-deficient crypts, indicating that apoptosis may be the major form of cell death (Fig. $5 \mathrm{~d}$ ). Taken together, our results suggest that Ufbp1 is crucial for survival of Paneth and goblet cells.

\section{Depletion of Ufbp1 resulted in activation of the UPR and cell death program in IECs}

A previous study indicated that depletion of the Ufm1 components rendered pancreatic $\beta$-cells susceptible to ER stress-induced apoptosis ${ }^{41}$. We have shown that the $\mathrm{Ufm} 1$ system is upregulated by ER stress ${ }^{42}$, and ablation of either Ufl1 or Ufbp1 results in elevated ER stress and activation of UPR in hematopoietic cells ${ }^{21,22}$. Therefore, the Ufm1 system appears to be crucial for ER homeostasis and cellular response to ER stress. In the intestine, acute deletion of $U f b p 1$ ( $L f b p 1^{f f f}$ :ROSA-CreERT2 treated with tamoxifen) led to upregulation of Grp78 and a set of cell death genes (Fig. 6a). Elevation of ER stress, indicated by increased staining of Grp78, appeared to occur in all types of IECs (Fig. 6b). In Paneth cells, Ufbp1 deficiency caused swelling of the ER network (Fig. 6c) and vacuolation of some ER structure (Fig. 6c, high magnification). We further examined activation of downstream signaling branches of the UPR. As shown in Fig. 6d, e, either acute or IEC-specific ablation of Ufbp1 caused slight increase of IRE1 $\alpha$ protein and significant accumulation of Xbp-1s, indicating that the IRE1-Xbp-1 branch of UPR was robustly activated by $U f b p 1$ deficiency (Fig. $6 \mathrm{~d}$, e). We also observed a substantial increase of total and active PERK in Ufbp 1 knockout IECs, while phosphorylation of eIF $2 \alpha$ was slightly increased in the acute deletion model ( $L f b p{ }^{f f f}$ : ROSA-CreERT2 mice treated with tamoxifen, Fig. 6d) but not significantly changed in the IEC-specific knockout model (Fig. 6e). Being consistent with our previous study, this result demonstrates the crucial role of the ufmylation pathway in modulating ER homeostasis.

\section{Chemical chaperone TUDCA alleviates loss of Ufbp 1- deficient Paneth cells}

To further evaluate the consequence of elevated ER stress and UPR activation in Ufbp1-deficient IECs, we 


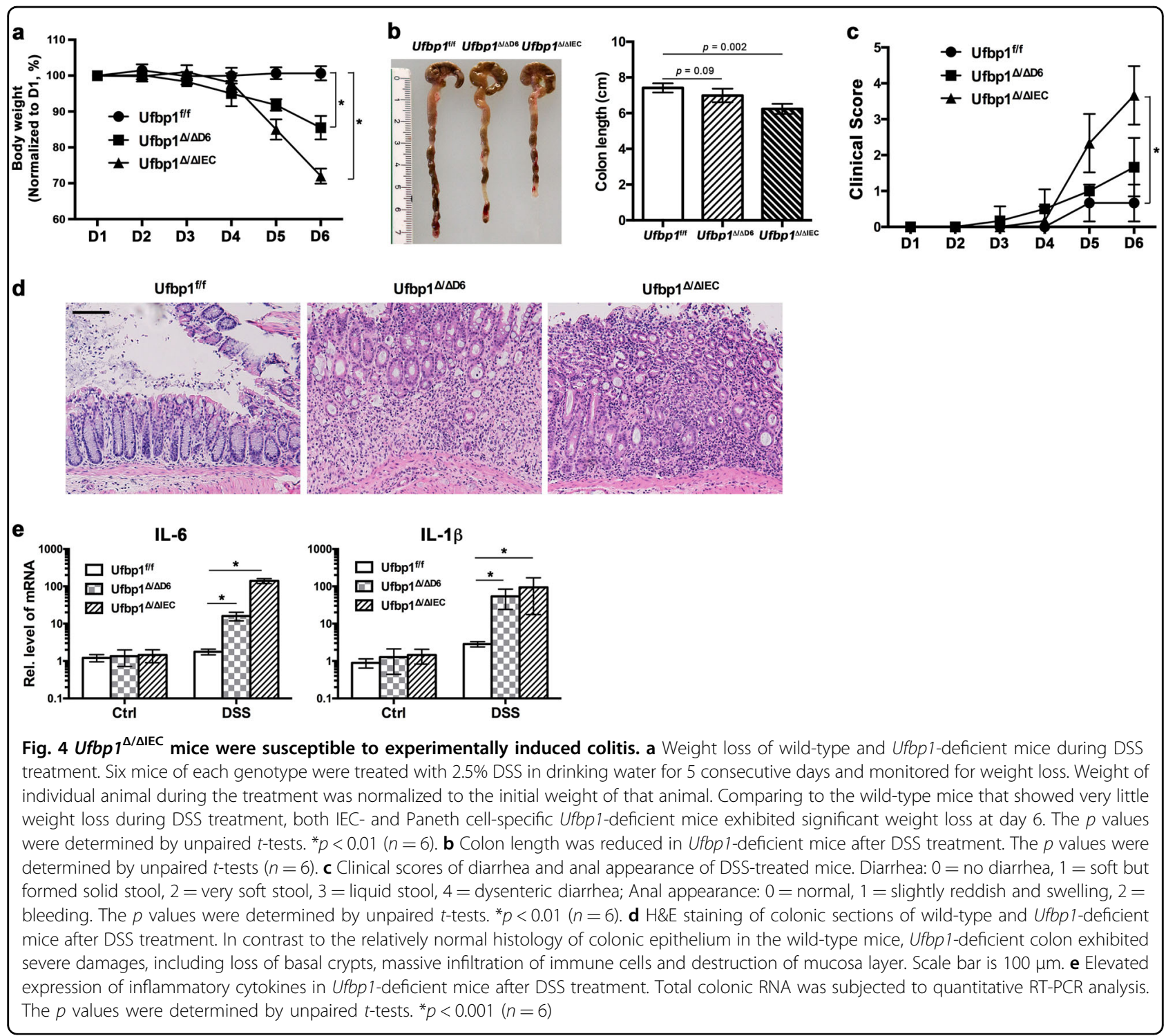

tested the effect of tauroursodeoxycholic acid (TUDCA), a natural bile salt that mitigates ER stress, in the acute knockout model ${ }^{43-45}$. As indicated by the Paneth cell number and gene expression, TUDCA treatment significantly alleviated loss of Paneth cells in Ufbp1-deficient crypts (Fig. 7a-d). Moreover, TUDCA partially suppressed Ufbp1 deletion-induced upregulation of Grp78 (Fig. 7e), cell death (Fig. 7f) and elevated expression of cell death genes (Fig. 7g). Together, these results suggest that elevated ER stress contributes to loss of $U f b p 1$-deficient Paneth cells.

\section{Discussion}

Recent genetic studies using knockout mouse models have established the indispensable role of the ufmylation pathway in embryonic development and hematopoiesis $^{20-22}$. Yet its physiological functions in other tissues and organs have not been defined. In this study, we report that both Ufl1 and Ufbp1, two key components of a Ufm1-specific E3 ligase, are highly expressed in intestinal exocrine cells and essential for their development and survival. Acute ablation of $U f l 1$ resulted in significant loss of Paneth and goblet cells, while IEC-specific Ufbp 1 knockout mice exhibited the same phenotype. Loss of Paneth cells in $U f b p 1^{\Delta / \Delta I E C}$ mice resulted in increased susceptibility to DSS-induced colitis. At the cellular and molecular levels, Ufbp1 deficiency led to elevated ER stress and activation of UPR, which in turn caused apoptosis of Paneth and goblet cells. Administration of a small chemical chaperone TUDCA partially prevented loss of Paneth cells in Ufbp1-deficient crypts. Taken together, our results have demonstrated a pivotal role of Ufl1 and Ufbp1 in development and survival of exocrine cell lineage and maintenance of intestinal homeostasis. 


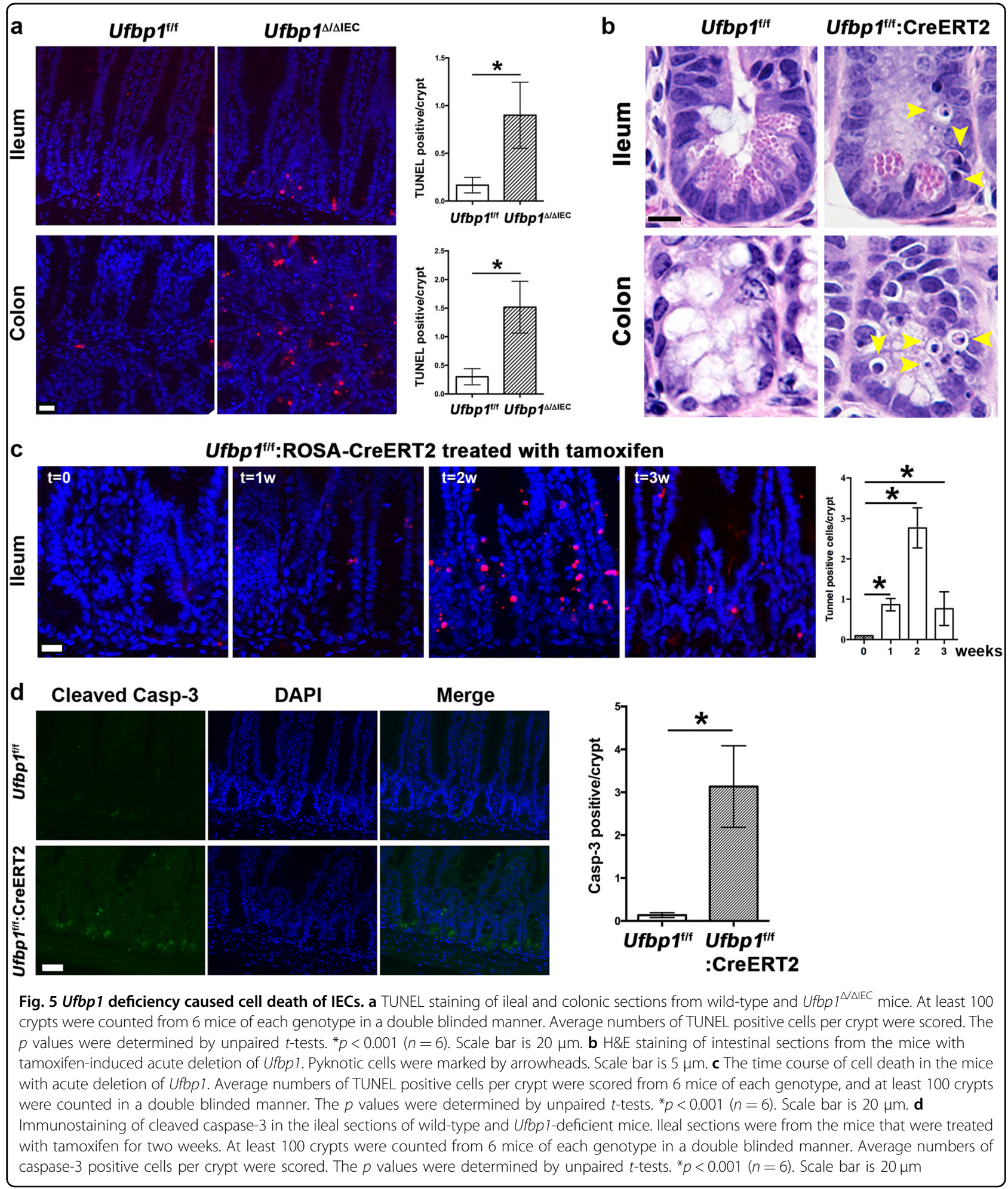

Intestinal exocrine cells including Paneth and goblet cells are responsible for production and secretion of a large amount of proteins that are essential for intestinal homeostasis and mucosal immunity. At the cellular level, both Paneth and goblet cells contain a rich rough ER network to cope with the demand for protein synthesis, modification, folding and secretion. Therefore, these cells are much more vulnerable to any perturbation of ER homeostasis and elevation of ER stress, a condition which is usually a result of accumulation of unfolded and 
a

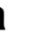

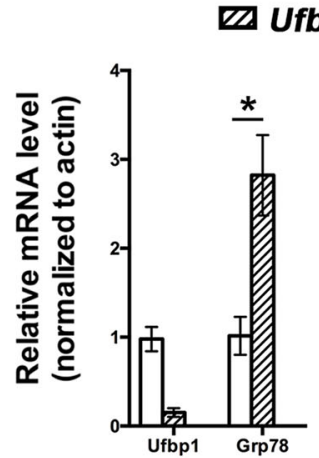

b

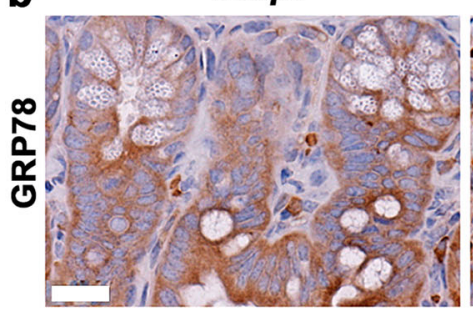

C
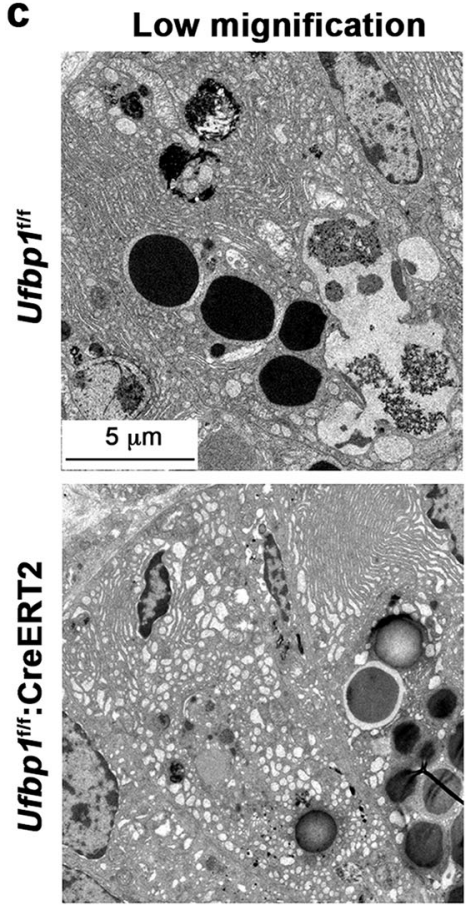

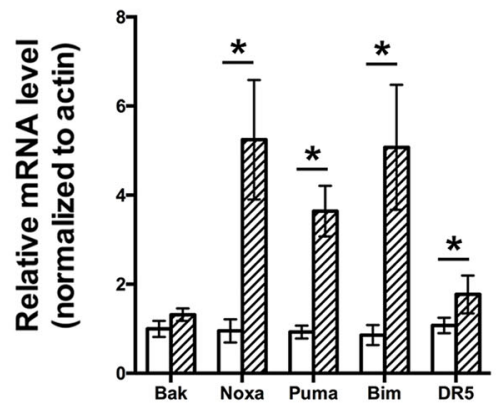

Ufbp 1 ${ }^{\text {fff }}:$ CreERT2
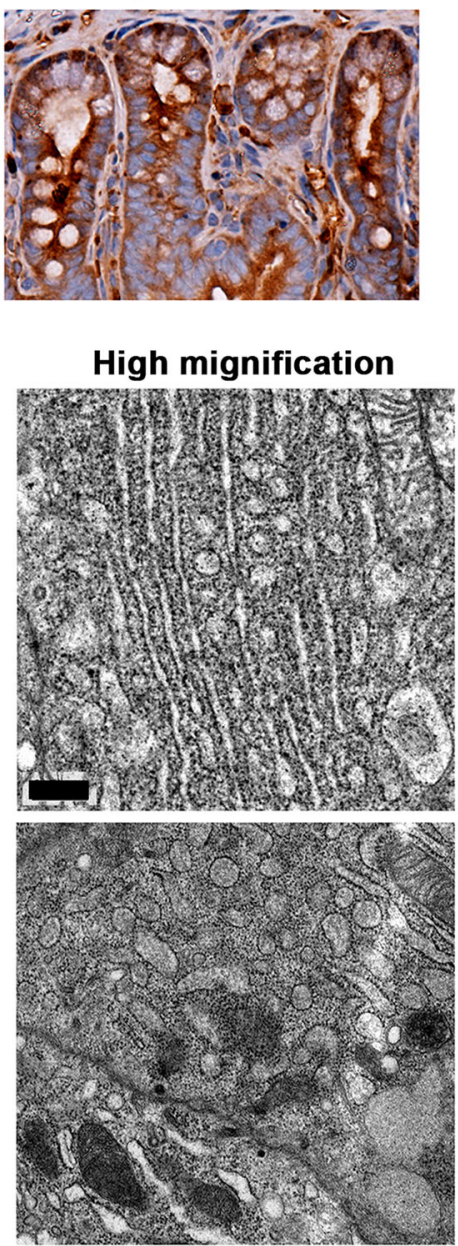

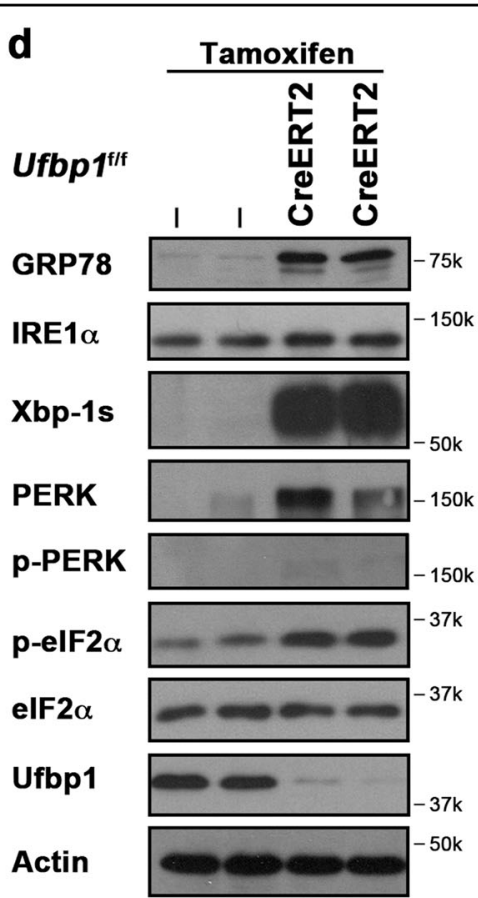

e
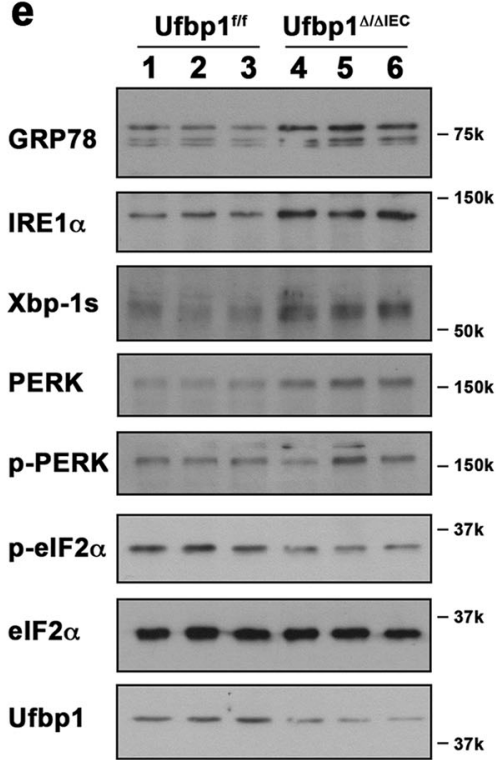

Actin

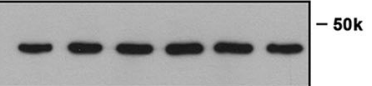

Fig. 6 Activation of the UPR and cell death program in Ufbp1-deficient IECs. a Upregulation of Grp78 and cell death genes induced by acute ablation of Ufbp1. Mice were treated with tamoxifen for 5 days and then killed at 2 weeks after initial treatment. Total RNA was isolated from ileal crypts and subject to quantitative RT-PCR analysis. The $p$ values were determined by unpaired $t$-tests. ${ }^{*} p<0.05(n=6)$. $\mathbf{b}$ Immunohistochemistry of Grp78 protein in the small intestinal sections. Scale bar is $20 \mu \mathrm{m}$. c Representative EM micrographs of Paneth cells in wild-type and tamoxifen-treated Ufbp 1 floxed mice (1-week treatment). Swelling and vacuolation of the ER structure was observed in Ufbp 1-deficient Paneth cells. Scale bar is $5 \mu \mathrm{m}$ for low magnification, and $0.5 \mu \mathrm{m}$ for high magnification. $\mathbf{d}$ Activation of UPR pathways in the mice with acute deletion of Ufbp 1. e UPR activation in the $U f b p 1^{\Delta \Delta \Delta E C}$ intestine. Crypts were isolated, lysed, and subjected to immunoblotting of specific antibodies as indicated 


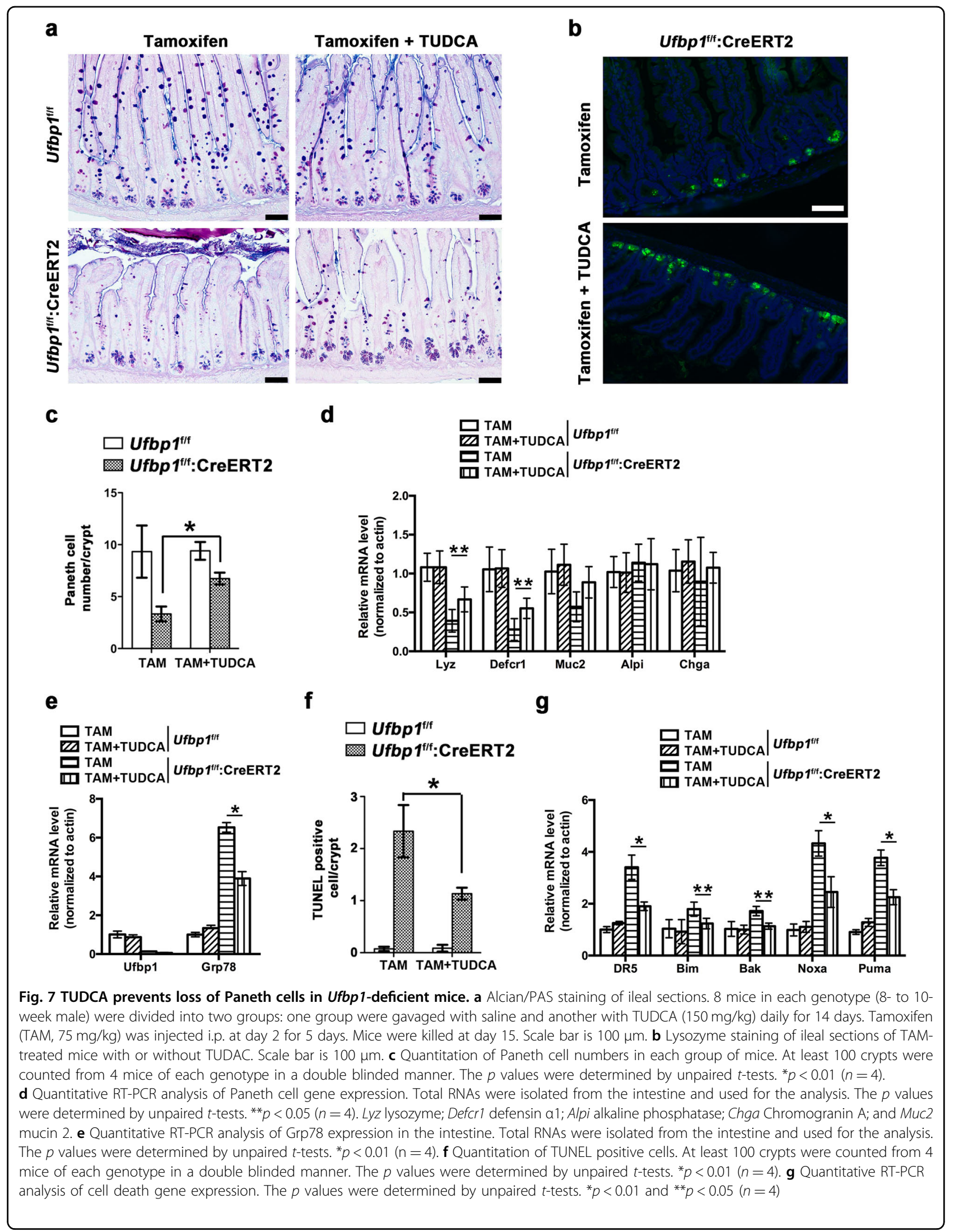


misfolded proteins in the ER. Many environmental factors, such as oxidative stress, viral infection, inflammation and nutrient deficiency, often cause elevation of ER stress in intestinal epithelial cells ${ }^{46,47}$. In response to elevated ER stress, cells activate the UPR, a highly conserved signaling pathway, to facilitate the folding of unfolded or misfolded proteins and restoration of ER homeostasis. Given its important role in maintaining proper ER function, it is not surprising that intestinal exocrine cells, such as Paneth and goblet cells whose main function is to synthesize and secrete a large quantity of proteins, are highly dependent on the functional UPR. Any disturbance or disruption of the UPR may cause severe reduction or even loss of their secretory capacity, which in turn leads to dysbiotic intestine and inflammatory diseases. Consistent with this notion, IBD patients were reported to have increased ER stress in ileul and/or colonic epithelia ${ }^{48-50}$. Furthermore, genome-wide association study (GWAS) and deep sequencing of IBD patients has identified several UPRrelated genes as IBD susceptibility loci. Rare variants of $X b p-1$ were identified as a risk locus for both ulcerative colitis and Crohn's disease, and its deficiency in mouse intestine led to complete loss of Paneth cells and partial loss of goblet cells, thereby resulted in increased susceptibility to DSS-induced colitis ${ }^{51}$. In addition, key UPR molecules such as IRE1 and eIF2 $\alpha$ also have a critical role in epithelial integrity and intestinal homeostasis. IRE1 $\beta$ knockout caused aberrant accumulation of mucin 2 mRNA and protein, resulting in ER distension, elevated $E R$ stress and barrier function defect ${ }^{52}$. IEC-specific ablation of IRE1 $\alpha$ leads to loss of goblet cells and spontaneous colitis, and IRE1 $\alpha^{-/-}$mice are more susceptible to DSS-induced colitis and ER stress-related apoptosis ${ }^{53}$. Phosphorylation of eIF2 $\alpha$, a key step in the UPR to attenuate protein translation, is essential for Paneth cell function, and the mice with loss of eIF $2 \alpha$ phosphorylation are more susceptible to Salmonella infection and experimentally induced colitis ${ }^{54}$. Deficiency of AGR2, an ERresided disulfide isomerase, also disrupted intestinal tissue homeostasis and caused development of spontaneous ileitis and colitis in mice ${ }^{55,56}$.

Our current study has identified the Ufm1 conjugation system, a metazoan-specific ubiquitination-like system, as a novel regulator of intestinal homeostasis. Mouse genetic studies have demonstrated its indispensable role in embryonic development, yet the working mechanism remains poorly understood at the cellular level. Emerging evidence from recent studies suggest its crucial role in maintaining ER homeostasis. Ufl1 and Ufbp1 are ERanchored proteins, and other components of ufmylation are also mainly associated with the $\mathrm{ER}^{14,16}$. In addition to ubiquitous expression of ufmylation-related genes in many cell types and tissues, we found that the components of the Ufm1 system, including Ufl1 and Ufbp1, were highly expressed in intestinal exocrine cells that contain very rich ER structure (Fig. 1a). Additionally, Ufm1 was reported to be a target of secretory cell-specific transcription factor MIST1 $1^{57}$, while several ufmylation-related genes are transcriptionally regulated by Xbp-1, a key transcription factor of the UPR ${ }^{42}$. The expression profile of these ufmylation genes is consistent with their functional role in the cells with rich ER structure. Deficiency of ufmylation genes such as Uba5 and Ufm1 led to accumulation and expansion of ER membranes ${ }^{30,42}$. Furthermore, overexpression of the components of ufmylation protects pancreatic beta cells and macrophages from ER stress-induced cell death ${ }^{41,58}$. Our previous and current studies have demonstrated that knockout of either Ufl1 or Ufbp 1 resulted in elevated ER stress and activation of the UPR in various types of cells, including hematopoietic and intestinal cells $\left({ }^{21,22}\right.$ and the results in this manuscript). Intriguingly, our findings were consistent with the results of recent studies conducted by DeJesus et al. $^{59}$ and Terai et al. ${ }^{60}$ In genome-wide CRISPR/ cas9 screenings, knockout of the ufmylation genes triggered the UPR activation in multiple cancer cell lines ${ }^{59,60}$. Taken together, the recent findings strongly suggest that the ufmylation pathway is a novel mechanism to regulate ER homeostasis and function, and deficiency of ufmylation may cause perturbation of ER homeostasis and elevated ER stress that leads to apoptosis of exocrine cells.

The next question is how ufmylation is engaged in the maintenance of ER homeostasis. One possible scenario is that the ufmylation pathway may modulate the activity of the key UPR player(s) in a direct manner. Liu et al. ${ }^{61}$ reported that Ufbp1 can directly interact and stabilize IRE1 $\alpha$, and knockdown of Ufbp1 resulted in IRE1 $\alpha$ degradation and attenuation of the IRE $1 \alpha-\mathrm{Xbp} 1$ signaling, which in turn activated the PERK signaling. However, in contrast to their findings, we found that $U f b p 1$ deficiency led to IRE1 $\alpha$ upregulation and robust Xbp-1 activation (Fig. 6). Our finding is in agreement with the study by Terai et al. ${ }^{60}$, showing that CRISPR/Cas9-mediated knockout of ufmylation genes in lung cancer cells specifically activated the IRE1 $\alpha$-Xbp1 branch. Apparently, more work is needed to establish the molecular $\operatorname{link}(\mathrm{s})$, either direct or indirect, between the ufmylation and UPR pathways. Another scenario is that the ufmylation pathway may be involved in controlling ER-related functions such as protein synthesis, modification, folding and secretion, and its deficiency may lead to accumulation of unfolded or misfolded protein, thereby resulting in ER stress elevation and UPR activation. Recently, Simsek et al. ${ }^{62}$ reported that Ufl1 was one of ribosome-associated proteins, and several ribosomal subunits and elongation factor eIF6 were potential Ufm1 targets. This finding indicates a potential role of ufmylation in control of protein translation. Interestingly enough, protein 
translation inhibitor cycloheximide can promote Ufm1 conjugation in tissue culture cells ${ }^{41,42}$. We speculate that the ufmylation pathway may represent a novel generic mechanism to co-ordinate protein translation and protein quality control in the ER. Further study, especially identification and characterization of Ufm 1 targets and their interacting proteins, is needed to test this hypothesis. In summary, our current work uncovered a crucial link between the ufmylation pathway and intestinal homeostasis. These findings may provide a basis for identification of novel therapeutic targets for treatment of IBD and other gastrointestinal diseases.

\section{Materials and methods}

\section{Ufl1 and Ufbp1 knockout mice and experimental} procedures

Ufl1 and Ufbp1 knockout mouse lines and genotyping methods were described in our previous reports ${ }^{21,22}$. Villin-Cre transgenic mouse line was originally from Dr. Sylvie Robine's laboratory ${ }^{38}$. Paneth cell-specific Defa6Cre transgenic line was obtained from Dr. Richard Blumberg's laboratory ${ }^{6}$. All mice were maintained in C57BL/6 background. Cre-mediated acute deletion of Ufl1 or Ufbp1 was induced by tamoxifen administration. Tamoxifen $(20 \mathrm{mg} / \mathrm{ml}$ in corn oil, Sigma, St. Louis, MO) was administered by 5-day intraperitoneal (IP) injection with an approximate dose of $75 \mathrm{mg}$ tamoxifen $/ \mathrm{kg}$ body weight. At two days prior to tamoxifen injection, tauroursodeoxycholic acid (TUDCA, $150 \mathrm{mg} / \mathrm{kg}$, Cayman Chemical, Ann Arbor, MI) was administrated daily by gavage, and the treatment was then continued through the course of experiment. Colitis-grade dextran sulfate sodium (DSS, MW 36,000-50,000) was purchased from MP Biomedicals (Santa Ana, CA). Mice were fed with $2.5-5 \%$ DSS in the drinking water for 5 days. Mice were housed in the animal facility at Augusta University, and all animal procedures were approved by IACUC of Augusta University.

Isolation and in vitro organoid culture of intestinal crypts

Crypts were isolated from the ileum of small intestine according to Sato et al. $^{63}$ with minor modifications. Briefly, the ileum of small intestine was harvested and opened lengthwise, and then washed multiple times with cold PBS. After removal of the villi with a cover glass, the intestine was cut into 2-3 large pieces, and then washed with cold PBS $(10-20 \mathrm{ml})$ for more than 10 times. Subsequently the fragments were incubated in $25 \mathrm{ml}$ of $2 \mathrm{mM}$ EDTA on ice for $15 \mathrm{~min}$ with a gentle shaking. After removal of the supernatant, the tissue was washed with cold PBS and then pipetted up and down 3-5 times. Released crypts were passed through a $70 \mu \mathrm{M}$ cell strainer and collected by 3 -min centrifugation at $100 \times g$. Isolated crypts were counted and resuspended in Growth Factor
Reduced Matrigel (Corning Life sciences, Corning, NY) and cultured in the complete medium: Advanced DMEM/ F12 supplemented with $1 \times \mathrm{B} 27,1 \times \mathrm{N} 2$ (Thermo Fisher Scientific, Waltham, MA), $1 \mathrm{mM} N$-acetyle-L-cysteine, HEPES (10 mM, pH 7.4), murine EGF (50 ng/ml, PeproTech, Rocky Hill, NJ), murine Noggin $(100 \mathrm{ng} / \mathrm{ml}$, PeproTech), 1/10th volume of R-Spondin-1 conditional medium (Trevigen, Gaitherburg, MD), 1×GlutaMax, $1 \times$ Penicillin/ Streptomycin (Thermo Fisher Scientific), and $100 \mu \mathrm{g} / \mathrm{ml}$ Normocin (Invivogen, San Diego, CA),

\section{Histology, immunohistochemistry, immunofluorescent staining, and immunoblotting}

HE and PAS/Alcian blue staining was performed by the Histology core of Augusta University, while Transmission Electron Microscope (TEM) was conducted by the EM core of Augusta University according to the standard procedures $^{22}$. Immunohistochemistry, immunofluorescent staining and Immunoblottings were performed as described previously ${ }^{22}$. Bright field and Epifluorescence images were obtained using Zeiss Observer D1 with AxioVision 4.8 software (Carl Zeiss Microscopy GmbH, Jena, Germany) and Keyence BZX700 fluorescent microscope with its corresponding software (Keyence America, Itasca, IL, USA).

The antibodies used in this study included: Ufl1 and Ufbp1 rat polyclonal antibodies (Li lab), Ufbp1 rabbit polyclonal antibody (21445-1-AP, Proteintech, Rosemont, IL), eIF2 $\alpha$ (\#5324, Cell Signaling, Danvers, MA), phosphoS51-eIF2 $\alpha$ (\#3398, Cell Signaling), Lysozyme (A0099, Agilent Dako, Santa Clara, CA), Grp78/Bip (\#3177, Cell Signaling), IRE1 $\alpha$ (\#3294, Cell Signaling), Xbp-1s (\#619502, Poly6195, BioLegend, San Diego, CA), PERK (\#3192, Cell Signaling), phospho-PERK (Thr980) (\#3197, Cell Signaling), cleaved caspase-3 (Asp175) (\#9664, Cell Signaling), Chromogranin A (Ab15160, Abcam, Cambridge, MA) and $\beta$-Actin (\#3700, Cell Signaling). All affinity-purified and species-specific HRP- and fluorophore-conjugated secondary antibodies were obtained from Jackson ImmunoResearch (West Grove, PA).

\section{TUNEL staining}

TUNEL staining was performed using in situ cell death detection kit (TMR Red, Roche, Basel, Switzerland) according to the manufacturer's instruction.

\section{Quantification of gut microbiota using 16s rRNA profiling}

Quantification of gut microbiota was performed according to Sivaprakasm et al. ${ }^{64}$ Feces $(\sim 100 \mathrm{mg})$ were suspended with $710 \mu \mathrm{l}$ of disruption buffer $(200 \mathrm{~mm} \mathrm{NaCl}$, $200 \mathrm{~mm}$ Tris pH 8.0, $20 \mathrm{~mm}$ EDTA pH 8.0 and 6\% SDS) and $500 \mu \mathrm{l}$ of phenol/chloroform/isoamyl alcohol, $\mathrm{pH} 8.0$ inside tubes containing Zirconium beads $(0.1 \mathrm{~mm}$ diameter, Benchmark Scientific, Edison, NJ, USA). The 
mixture was homogenized using a Beadbeater (BioSpec, Bartlesville, OK, USA) for 2 cycles of 2 min each. Sample was centrifuged at $7000 \mathrm{~g}$ for $3 \mathrm{~min}$ and aqueous phase was collected and a second round of phenol/chloroform/ isoamyl extraction was performed. DNA from the clear aqueous phase was precipitated using sodium acetate and isopropanol. Dried DNA pellet was dissolved in TE buffer. Quantitative PCR using group-specific PCR primers were used to quantify relative abundance of different groups to the total gut microbiota.

\begin{tabular}{lll}
\hline Target & Forward primer & Reverse primer \\
\hline All bacteria & ACTCCTACGGGAGGCAGCAGT & GTATTACCGCGGCTGCT \\
& & GGCAC \\
Bifidobacterium & TCGCGTCYGGTGTGAAA & CCACATCCAGCRTCCAC \\
Prevotellaceae & CCAGCCAAGTAGCGTGCA & TGGACCTTCCGTATTACC \\
\hline
\end{tabular}

\section{Quantitative real-time PCR}

Total RNA from each sample was isolated with the GeneJET RNA Purification Kit (Thermo Fisher Scientific) or TRIzol reagent (Thermo Fisher Scientific), and then reversely transcribed using AMV reverse transcriptase and random primers according to the manufacturer's instruction (Thermo Fisher Scientific). Quantitative RTPCR was performed using the iTaq Universal SYBR Green Supermix kit (BIO-RAD, Hercules, CA) with 40 cycles of $95^{\circ} \mathrm{C}$ for $15 \mathrm{~s}$ and $60^{\circ} \mathrm{C}$ for $1 \mathrm{~min}$ on StepOnePlus RealTime PCR System (Thermo Fisher Scientific). The results were analyzed by StepOne Software (Version 2.1, Life Technologies). Relative expression level of each transcript was normalized to murine beta-actin by using the $2^{(- \text {delta }}$ delta Ct) method. The following is the list of primers used in this study:

\begin{tabular}{|c|c|c|}
\hline Gene (mouse) & Forward primer & Reverse primer \\
\hline Actin & GACCTCTATGCCAACACAGT & AGTACTTGCGCTCAGGAGGA \\
\hline $\begin{array}{l}\text { Ufl1 (exon7- } \\
\text { specific) }\end{array}$ & CCAGTGAACTCTITGGTITCAA & CCATTCTGCCTGAAAAAGGA \\
\hline $\begin{array}{l}\text { Ufbpl (exon3,4- } \\
\text { specific) }\end{array}$ & GAAGCCAGCAGAAGTTCACC & GAAGCCGTTCCTCTTCCTTC \\
\hline Grp78/Bip & ACTTGGGGACCACCTATTCCT & ATCGCCAATCAGACGCTCC \\
\hline Bak & AAAATGGCATCTGGACAAGG & AAGATGCTGTTGGGTTCCAG \\
\hline Noxa & GGCAGAGCTACCACCTGAGT & TTGAGCACACTCGTCCTTCA \\
\hline Puma & GCCCAGCAGCACTTAGAGTC & TGTCGATGCTGCTCTTCTTG \\
\hline Bim & TGCAGAGGATGATTGCTGAC & AGTACTTGCGCTCAGGAGGA \\
\hline DR5 & TGACTACACCAGCCATTCCA & AGTTCCTCTTCCCCGTCAGT \\
\hline Lysozyme 2 & ATGGAATGGCTGGCTACTATGG & ACCAGTATCGGCTATTGATCTGA \\
\hline Bim & TGCAGAGGATGATTGCTGAC & AGTACTTGCGCTCAGGAGGA \\
\hline Defcrl & $\begin{array}{l}\text { AAGAGACTAAAACTGAGGAGC } \\
\text { AGC }\end{array}$ & CGACAGCAGAGCGTGTA \\
\hline Defcr5 & $\begin{array}{l}\text { AGGCTGATCCTATCCACAAA } \\
\text { ACAG }\end{array}$ & TGAAGAGCAGACCCTTCTTGGC \\
\hline
\end{tabular}

continued

\begin{tabular}{lll}
\hline Gene (mouse) & Forward primer & Reverse primer \\
\hline $\begin{array}{l}\text { Intestinal alkaline } \\
\text { phosphatase }\end{array}$ & CACAGCTACCTGGCACTGA & GGTCTCTGACGACAGGGGTA \\
Chromogranin A & CCAATACCCAATCACCAACC & TIGTAGCCTGCATGGAAGTG \\
Mucin 2 & GCCTGTTGATAGCTGCTAT & GTTCCGCCAGTCAATGCAGACAC \\
\hline
\end{tabular}

\section{Statistical analysis}

All statistical analyses were performed using Graph Prism software. $p$ values were determined by unpaired $t$ tests between two set of data. A $p$ value $<0.05$ was considered to be significant.

\section{Acknowledgements \\ This work was supported by NIH/NIDDK (1R01DK113409) and NSFC (81770515)} to H.L., and NIH/NIDDK (DK088199) to R.B.

\section{Author details \\ ${ }^{1}$ College of Animal Science and Technology, Nanjing Agricultural University, 210095 Nanjing, Jiangsu, China. ${ }^{2}$ Department of Biochemistry \& Molecular Biology, Georgia Cancer Center, Medical College of Georgia, Augusta University, Augusta, GA 30912, USA. ${ }^{3}$ Department of Stomatology, Tongji Hospital, Tongji Medical College, Huazhong University of Science and Technology, 430030 Wuhan, Hubei, China. ${ }^{4}$ Department of Biochemistry, Faculty of Basic Medicine, Nanchang University, 330006 Nanchang, Jiangxi, China. ${ }^{5}$ Division of Gastroenterology, Department of Medicine, Brigham and Women's Hospital, Harvard Medical School, Boston, MA 02115, USA. ${ }^{6}$ The 10th People's Hospital, Tongji University, Shanghai, China}

\section{Author contributions}

Y.C., G.Z., S.L., Z.P., M.Q., C.P., C.L., H.Z., and B.I. contribute to acquiring and analyzing the data; Y.C., J.v.R., D.B., K.L., R.B., N.S., and H.L. contribute to designing the work and analyzing the data; Y.C. and H.L. contribute to drafting the manuscript; and all authors contribute to commenting and revising the manuscript.

\section{Conflict of interest}

The authors declare that they have no conflict of interest.

\section{Publisher's note}

Springer Nature remains neutral with regard to jurisdictional claims in published maps and institutional affiliations.

Supplementary Information accompanies the paper at (https://doi.org/ 10.1038/s41421-018-0070-x).

Received: 13 June 2018 Revised: 29 October 2018 Accepted: 30 October 2018

Published online: 29 January 2019

\footnotetext{
References

1. McCauley, H. A. \& Guasch, G. Three cheers for the goblet cell: maintaining homeostasis in mucosal epithelia. Trends Mol. Med. 21, 492-503 (2015).

2. Bevins, C. L. \& Salzman, N. H. Paneth cells, antimicrobial peptides and maintenance of intestinal homeostasis. Nat. Rev. Microbiol. 9, 356-368 (2011).

3. Clevers, H. C. \& Bevins, C. L. Paneth cells: maestros of the small intestinal crypts. Annu. Rev. Physiol. 75, 289-311 (2013).

4. Sato, T. et al. Paneth cells constitute the niche for Lgr 5 stem cells in intestinal crypts. Nature 469, 415-418 (2011).
} 
5. Yilmaz, O. H. et al. mTORC1 in the Paneth cell niche couples intestinal stemcell function to calorie intake. Nature 486, 490-495 (2012).

6. Adolph, T. E. et al. Paneth cells as a site of origin for intestinal inflammation Nature 503, 272-276 (2013).

7. Liu, T. C. et al. Paneth cell defects in Crohn's disease patients promote dysbiosis. JCl Insight 1, e86907 (2016).

8. Velcich, A. et al. Colorectal cancer in mice genetically deficient in the mucin Muc2. Science 295, 1726-1729 (2002).

9. McGuckin, M. A., Eri, R. D., Das, l., Lourie, R. \& Florin, T. H. Intestinal secretory cell ER stress and inflammation. Biochem. Soc. Trans. 39, 1081-1085 (2011).

10. Kerscher, O., Felberbaum, R. \& Hochstrasser, M. Modification of proteins by ubiquitin and ubiquitin-like proteins. Annu. Rev. Cell. Dev. Biol. 22, 159-180 (2006).

11. Komatsu, M. et al. A novel protein-conjugating system for Ufm1, a ubiquitinfold modifier. EMBO J. 23, 1977-1986 (2004).

12. Sasakawa, H. et al. Solution structure and dynamics of Ufm1, a ubiquitin-fold modifier 1. Biochem. Biophys. Res. Commun. 343, 21-26 (2006).

13. Kwon, J. et al. A novel LZAP-binding protein, NLBP, inhibits cell invasion. J. Biol. Chem. 285, 12232-12240 (2010).

14. Wu, J., Lei, G., Mei, M., Tang, Y. \& Li, H. A novel C53/LZAP-interacting protein regulates stability of C53/LZAP and DDRGK domain-containing Protein 1 (DDRGK1) and modulates NF-kappaB signaling. J. Biol. Chem. 285, 15126-15136 (2010).

15. Shiwaku, H. et al. Suppression of the novel ER protein Maxer by mutant ataxin1 in Bergman glia contributes to non-cell-autonomous toxicity. EMBO J. 29, 2446-2460 (2010).

16. Tatsumi, K. et al. A novel type of E3 ligase for the Ufm1 conjugation system. J. Biol. Chem. 285, 5417-5427 (2010).

17. Neziri, D. et al. Cloning and molecular characterization of Dashurin encoded by C20orf116, a PCl-domain containing protein. Biochim. Biophys. Acta 1800, 430-438 (2010).

18. Lee, J. W., Choi, H. S., Gyuris, J., Brent, R. \& Moore, D. D. Two classes of proteins dependent on either the presence or absence of thyroid hormone for interaction with the thyroid hormone receptor. Mol. Endocrinol. 9, 243-254 (1995).

19. Yoo, H. M. et al. Modification of ASC1 by UFM1 is crucial for ERalpha transactivation and breast cancer development. Mol. Cell 56, 261-274 (2014).

20. Tatsumi, K. et al. The Ufm1-activating enzyme Uba5 is indispensable for erythroid differentiation in mice. Nat. Commun. 2, 181 (2011).

21. Zhang, M. et al. RCAD/Ufl1, a Ufm1 E3 ligase, is essential for hematopoietic stem cell function and murine hematopoiesis. Cell Death Differ. 22, 1922-1934 (2015).

22. Cai, Y. et al. UFBP1, a key component of the Ufm1 conjugation system, is essential for ufmylation-mediated regulation of erythroid development. PLoS Genet. 11, e1005643 (2015).

23. Tanaka, Y. et al. Genome-wide association study identified ITPA/DDRGK1 variants reflecting thrombocytopenia in pegylated interferon and ribavirin therapy for chronic hepatitis C. Hum. Mol. Genet. 20, 3507-3516 (2011).

24. van Loon, J. et al. Genome-wide association studies identify genetic loci for low von Willebrand factor levels. Eur J. Hum. Genet. 24, 1035-1040 (2015).

25. Lu, H., Yang, Y., Allister, E. M., Wijesekara, N. \& Wheeler, M. B. The identification of potential factors associated with the development of type 2 diabetes: a quantitative proteomics approach. Mol. Cell. Proteom. 7, 1434-1451 (2008).

26. Azfer, A., Niu, J., Rogers, L. M., Adamski, F. M. \& Kolattukudy, P. E. Activation of endoplasmic reticulum stress response during the development of ischemic heart disease. Am. J. Physiol. Heart Circ. Physiol. 291, H1411-H1420 (2006).

27. Watson, C. M. et al. Identification of a mutation in the ubiquitin-fold modifier 1-specific peptidase 2 gene, UFSP2, in an extended South African family with Beukes hip dysplasia. S. Afr. Med. J. 105, 558-563 (2015).

28. Pang, Q. et al. UFM1 protects macrophages from oxLDL-induced foam cell formation through a liver $X$ receptor alpha dependent pathway. J. Atheroscler. Thromb. 22, 1124-1140 (2015).

29. Maran, S. et al. Gastric precancerous lesions are associated with gene variants in Helicobacter pylori-susceptible ethnic Malays. World J. Gastroenterol. 19, 3615-3622 (2013).

30. Colin, E. et al. Biallelic variants in UBA5 reveal that disruption of the UFM1 cascade can result in early-onset encephalopathy. Am. J. Hum. Genet. 99, 695-703 (2016).

31. Duan, $R$. et al. UBA5 mutations cause a new form of autosomal recessive cerebellar ataxia. PLoS ONE 11, e0149039 (2016).
32. Muona, M. et al. Biallelic variants in UBA5 Link Dysfunctional UFM1 Ubiquitinlike Modifier Pathway to Severe Infantile-Onset Encephalopathy. Am. J. Hum Genet. 99, 683-694 (2016).

33. Arnadottir, G. A. et al. Compound heterozygous mutations in UBA5 causing early-onset epileptic encephalopathy in two sisters. BMC Med. Genet. 18, 103 (2017).

34. Mignon-Ravix, C. et al. Abnormal function of the UBA5 protein in a case of early developmental and epileptic encephalopathy with suppression-burst. Hum. Mutat. 39, 934-938 (2018).

35. Nahorski, M. S. et al. Biallelic UFM1 and UFC1 mutations expand the essential role of ufmylation in brain development. Brain 141, 1934-1945 (2018).

36. Nalls, M. A. et al. Large-scale meta-analysis of genome-wide association data identifies six new risk loci for Parkinson's disease. Nat. Genet. 46, 989-993 (2014).

37. Egunsola, A. T. et al. Loss of DDRGK1 modulates SOX9 ubiquitination in spondyloepimetaphyseal dysplasia. J. Clin. Invest. 127, 1475-1484 (2017).

38. el Marjou, F. et al. Tissue-specific and inducible Cre-mediated recombination in the gut epithelium. Genesis 39, 186-193 (2004).

39. Salzman, N. H. \& Bevins, C. L. Dysbiosis - a consequence of Paneth cell dysfunction. Semin. Immunol. 25, 334-341 (2013).

40. Zhang, Q. et al. Commensal bacteria direct selective cargo sorting to promote symbiosis. Nat. Immunol. 16, 918-926 (2015).

41. Lemaire, K. et al. Ubiquitin fold modifier 1 (UFM1) and its target UFBP1 protect pancreatic beta cells from ER stress-induced apoptosis. PLoS ONE 6, e18517 (2011).

42. Zhang, Y., Zhang, M., Wu, J., Lei, G. \& Li, H. Transcriptional regulation of the Ufm1 conjugation system in response to disturbance of the endoplasmic reticulum homeostasis and inhibition of vesicle trafficking. PLOS ONE 7, e48587 (2012).

43. Xie, Q. et al. Effect of tauroursodeoxycholic acid on endoplasmic reticulum stress-induced caspase-12 activation. Hepatology 36, 592-601 (2002).

44. Ozcan, U. et al. Chemical chaperones reduce ER stress and restore glucose homeostasis in a mouse model of type 2 diabetes. Science 313, 1137-1140 (2006).

45. Cao, S. S. et al. The unfolded protein response and chemical chaperones reduce protein misfolding and colitis in mice. Gastroenterology 144, 989-1000 (2013).

46. Kaser, A., Flak, M. B., Tomczak, M. F. \& Blumberg, R. S. The unfolded protein response and its role in intestinal homeostasis and inflammation. Exp. Cell Res. 317, 2772-2779 (2011).

47. Cao, S. S. Endoplasmic reticulum stress and unfolded protein response in inflammatory bowel disease. Inflamm. Bowel Dis. 21, 636-644 (2015).

48. Heazlewood, C. K. et al. Aberrant mucin assembly in mice causes endoplasmic reticulum stress and spontaneous inflammation resembling ulcerative colitis. PLoS Med. 5, e54 (2008).

49. Shkoda, A. et al. Interleukin-10 blocked endoplasmic reticulum stress in intestinal epithelial cells: impact on chronic inflammation. Gastroenterology 132, 190-207 (2007).

50. Treton, X. et al. Altered endoplasmic reticulum stress affects translation in inactive colon tissue from patients with ulcerative colitis. Gastroenterology $\mathbf{1 4 1}$ 1024-1035 (2011)

51. Kaser, A. et al. XBP1 links ER stress to intestinal inflammation and confers genetic risk for human inflammatory bowel disease. Cell 134, 743-756 (2008).

52. Bertolotti, A. et al. Increased sensitivity to dextran sodium sulfate colitis in IRE1 beta-deficient mice. J. Clin. Invest. 107, 585-593 (2001).

53. Zhang, H. S. et al. The endoplasmic reticulum stress sensor IRE1alpha in intestinal epithelial cells is essential for protecting against colitis. J. Biol. Chem. 290, 15327-15336 (2015).

54. Cao, S. S. et al. Phosphorylation of elF2alpha is dispensable for differentiation but required at a posttranscriptional level for paneth cell function and intestinal homeostasis in mice. Inflamm. Bowel Dis. 20, 712-722 (2014).

55. Park, S. W. et al. The protein disulfide isomerase AGR2 is essential for production of intestinal mucus. Proc. Natl Acad. Sci. USA 106, 6950-6955 (2009).

56. Zhao, F. et al. Disruption of Paneth and goblet cell homeostasis and increased endoplasmic reticulum stress in Agr2-/- mice. Dev. Biol. 338, 270-279 (2010).

57. Lo, H. G. et al. A single transcription factor is sufficient to induce and maintain secretory cell architecture. Genes Dev. 31, 154-171 (2017).

58. $\mathrm{Hu}, \mathrm{X}$. et al. Ubiquitin-fold modifier 1 inhibits apoptosis by suppressing the endoplasmic reticulum stress response in Raw264.7 cells. Int. J. Mol. Med. 33 1539-1546 (2014). 
59. DeJesus, R. et al. Functional CRISPR screening identifies the ufmylation pathway as a regulator of SQSTM1/p62. Elife 5, e17290 (2016).

60. Terai, $\mathrm{H}$. et al. ER stress signaling promotes the survival of cancer "persister cells" tolerant to EGFR tyrosine kinase inhibitors. Cancer Res. 78, 1044-1057 (2018).

61. Liu, J. et al. A critical role of DDRGK1 in endoplasmic reticulum homoeostasis via regulation of IRE1alpha stability. Nat. Commun. 8, 14186 (2017).
62. Simsek, D. et al. The mammalian Ribo-interactome reveals ribosome functional diversity and heterogeneity. Cell 169, 1051-1065 e1018 (2017).

63. Sato, T. et al. Single Lgr5 stem cells build crypt-villus structures in vitro without a mesenchymal niche. Nature 459, 262-265 (2009).

64. Sivaprakasam, S. et al. An essential role of Ffar2 (Gpr43) in dietary fibremediated promotion of healthy composition of gut microbiota and suppression of intestinal carcinogenesis. Oncogenesis 5, e238 (2016). 